\title{
DETERMINING A PARTICULAR SOLUTION FOR THE SYSTEMS OF LINEAR DIFFERENTIAL EQUATIONS WITH CONSTANT COEFFICIENTS
}

\author{
Vasile CĂRUȚAȘU \\ "Nicolae Bălcescu" Land Forces Academy, Sibiu, Romania \\ v.carutasu@yahoo.com
}

\begin{abstract}
As with the $n$-th order linear differential equations with constant coefficients, the problem to be solved is related to determining a particular solution, and then, using the general solution of the attached homogeneous system of linear differential equations with constant coefficients, to write the general solution of the initially given system. For homogeneous systems of linear differential equations with constant coefficients, the determination of the general solution is the method of eliminating or reducing which make the system a linear differential equation of the same order as that of the system, and its methods of solving it applies or the method of own values and vectors. If the system is non-homogeneous, then we also have to determine a particular solution that can be done in the same way as in the case of $n$-th order differential equations with constant coefficients, if the method of reduction or elimination was used, or the method of variation of constants, regardless of the method used to determine the general solution of the attached homogenous system of linear differential equations with constant coefficients. Whichever method is used, determining a particular solution for a system of linear differential equations with constant coefficients is difficult, in this study being proposed a method similar to that of $n$-th order linear differential equations with constant coefficients.
\end{abstract}

Keywords: systems of linear differential equations with constant coefficients, homogeneous systems of linear differential equations with constant coefficients, particular solution, general solution

\section{Introduction}

This study presents a simple and practical method to determine a particular solution for a system of linear differential equations with constant coefficients, and starts from the observation of how you can get a particular solution to an $n$-th order linear differential equation with constant coefficients.

The free terms of the system can be part of the same class of functions that the free term can be part of for the $n$-th order linear differential equations with constant coefficients, namely a combination such as: 


$$
\begin{aligned}
& P(x)+P_{1}(x) \cdot e^{\alpha_{1} \cdot x}+P_{2}(x) \cdot e^{\alpha_{2} \cdot x}+\ldots+P_{n}(x) \cdot e^{\alpha_{n} \cdot x}+ \\
& +e^{\omega_{1} \cdot x} \cdot\left(Q_{11}(x) \cdot \cos \left(\beta_{1} \cdot x\right)+Q_{12}(x) \cdot \sin \left(\beta_{1} \cdot x\right)\right)+ \\
& +e^{\omega_{2} \cdot x} \cdot\left(Q_{21}(x) \cdot \cos \left(\beta_{2} \cdot x\right)+Q_{22}(x) \cdot \sin \left(\beta_{2} \cdot x\right)\right)+\ldots+ \\
& +e^{\omega_{m} \cdot x} \cdot\left(Q_{m 1}(x) \cdot \cos \left(\beta_{m} \cdot x\right)+Q_{m 2}(x) \cdot \sin \left(\beta_{m} \cdot x\right)\right)+ \\
& +Q_{1}(x) \cdot \cos \left(\gamma_{1} \cdot x\right)+Q_{2}(x) \cdot \cos \left(\gamma_{2} \cdot x\right)+\ldots+Q_{k}(x) \cdot \cos \left(\gamma_{k} \cdot x\right)+ \\
& +R_{1}(x) \cdot \sin \left(\delta_{1} \cdot x\right)+R_{2}(x) \cdot \sin \left(\delta_{2} \cdot x\right)+\ldots+R_{l}(x) \cdot \sin \left(\delta_{l} \cdot x\right)+ \\
& +e^{\varepsilon_{1} \cdot x} S_{1}(x) \cdot \cos \left(\lambda_{1} \cdot x\right)+e^{\varepsilon_{2} \cdot x} S_{2}(x) \cdot \cos \left(\lambda_{2} \cdot x\right)+\ldots+e^{\varepsilon_{r} \cdot x} S_{r}(x) \cdot \cos \left(\lambda_{r} \cdot x\right)+ \\
& +e^{\rho_{1} \cdot x} T_{1}(x) \cdot \sin \left(\theta_{1} \cdot x\right)+e^{\rho_{2} \cdot x} T_{2}(x) \cdot \sin \left(\theta_{2} \cdot x\right)+\ldots+e^{\rho_{s} \cdot x} T_{s}(x) \cdot \sin \left(\theta_{s} \cdot x\right)
\end{aligned}
$$

where $\beta_{i} \neq \gamma_{j},(\forall) i \in \overline{1, m}$ and $j \in \overline{1, k}$,

$\beta_{i} \neq \delta_{j},(\forall) i \in \overline{1, m}$ and $j \in \overline{1, l}$,

$\beta_{i} \neq \lambda_{j},(\forall) i \in \overline{1, m}$ and $j \in \overline{1, r}$,

$\beta_{i} \neq \theta_{j},(\forall) i \in \overline{1, m}$ and $j \in \overline{1, s}$,

$\gamma_{i} \neq \delta_{j},(\forall) i \in \overline{1, k}$ and $j \in \overline{1, l}$,

$\gamma_{i} \neq \lambda_{j},(\forall) i \in \overline{1, k}$ and $j \in \overline{1, r}$,

$\gamma_{i} \neq \theta_{j},(\forall) i \in \overline{1, k}$ and $j \in \overline{1, s}$,

$\delta_{i} \neq \lambda_{j},(\forall) i \in \overline{1, l}$ and $j \in \overline{1, r}$,

$$
\begin{aligned}
& P_{1}(x) \cdot e^{\alpha_{1} \cdot x}+P_{2}(x) \cdot e^{\alpha_{2} \cdot x}+\ldots+P_{n}(x) \cdot e^{\alpha_{n} \cdot x}+ \\
& +e^{\gamma_{1} \cdot x} \cdot\left(Q_{1}(x) \cdot \cos \left(\beta_{1} \cdot x\right)+R_{1}(x) \cdot \sin \left(\beta_{1} \cdot x\right)\right)+ \\
& +e^{\gamma_{2} \cdot x} \cdot\left(Q_{2}(x) \cdot \cos \left(\beta_{2} \cdot x\right)+R_{2}(x) \cdot \sin \left(\beta_{2} \cdot x\right)\right)+\ldots+ \\
& +e^{\gamma_{m} \cdot x} \cdot\left(Q_{m}(x) \cdot \cos \left(\beta_{m} \cdot x\right)+R_{m}(x) \cdot \sin \left(\beta_{m} \cdot x\right)\right)
\end{aligned}
$$

$$
\begin{aligned}
& \delta_{i} \neq \theta_{j},(\forall) i \in \overline{1, l} \text { and } j \in \overline{1, s}, \\
& \lambda_{i} \neq \theta_{j},(\forall) i \in \overline{1, r} \text { and } j \in \overline{1, s},
\end{aligned}
$$$$
\text { and } P, P_{i}, i \in \overline{1, n}, Q_{i j}, i \in \overline{1, m} \text { and } j \in \overline{1,2} \text {, }
$$$$
Q_{i}, \quad i \in \overline{1, k}, \quad S_{i}, \quad i \in \overline{1, r}, \quad T_{i}, \quad i \in \overline{1, s} \text {, are }
$$
polynomials.

In the same way it can be shown that, without restricting the generality degree, the free terms of the system can be in the form:
This will be the form used for the free terms of the system we want to determine a particular solution to.

\section{Description of the problem}

We will consider that we have a system of linear differential equations with constant coefficients of the form

$$
\left\{\begin{array}{l}
y_{1}^{\prime}(x)=a_{11} \cdot y_{1}(x)+a_{12} \cdot y_{2}(x)+\ldots+a_{1 n} \cdot y_{n}(x)+f_{1}(x) \\
y_{2}^{\prime}(x)=a_{21} \cdot y_{1}(x)+a_{22} \cdot y_{2}(x)+\ldots+a_{2 n} \cdot y_{n}(x)+f_{2}(x) \\
\vdots \\
y_{n}^{\prime}(x)=a_{n 1} \cdot y_{1}(x)+a_{n 2} \cdot y_{2}(x)+\ldots+a_{n n} \cdot y_{n}(x)+f_{n}(x)
\end{array}\right.
$$

where $x \in I \subset \boldsymbol{R}$ is an interval of $\boldsymbol{R}$, and $f_{i}$, $i \in \overline{1, n}$, are functions like (2).
The attached homogeneous system of linear differential equations with constant coefficients will be like 


$$
\left\{\begin{array}{l}
y_{1}^{\prime}(x)=a_{11} \cdot y_{1}(x)+a_{12} \cdot y_{2}(x)+\ldots+a_{1 n} \cdot y_{n}(x) \\
y_{2}^{\prime}(x)=a_{21} \cdot y_{1}(x)+a_{22} \cdot y_{2}(x)+\ldots+a_{2 n} \cdot y_{n}(x) \\
\vdots \\
y_{n}^{\prime}(x)=a_{n 1} \cdot y_{1}(x)+a_{n 2} \cdot y_{2}(x)+\ldots+a_{n n} \cdot y_{n}(x)
\end{array}\right.
$$

where $x \in I \subset \boldsymbol{R}$ is an interval of $\boldsymbol{R}$.

The particular solution of the system of linear differential equations with constant coefficients (3), in which free terms $f_{i}(x)$, $i \in \overline{1, n}$ are functions in the form of (2) is constructed like the that of the $n$-th order linear differential equations with constant coefficients.

The characteristic equation attached to the homogeneous system of linear differential equations with constant coefficients (4) is of the form:

\section{The form of the particular solution and its determination exemplification}

As in the case of $n$-th order linear differential equations with constant coefficients, the construction of a particular solution is based on the characteristic equation attached to the homogeneous system (4).

$$
\left|\begin{array}{crrc}
a_{11}-\lambda & a_{12} & \ldots & a_{1 n} \\
a_{21} & a_{22}-\lambda & \ldots & a_{2 n} \\
& \vdots & & \\
a_{n 1} & a_{n 2} & \ldots & a_{n n}-\lambda
\end{array}\right|=0 .
$$

We will assume that it has the following expansion:

$$
\left|\begin{array}{cccc|c}
a_{11}-\lambda & a_{12} & \ldots & a_{1 n} & (-1)^{n} \cdot\left(\lambda-a_{1}\right)^{n_{1}} \cdot\left(\lambda-a_{2}\right)^{n_{2}} \cdot \ldots \cdot\left(\lambda-a_{q}\right)^{n_{q}} \\
a_{21} & a_{22}-\lambda & \ldots & a_{2 n} \\
& \vdots & & \\
a_{n 1} & a_{n 2} & \ldots & a_{n n}-\lambda
\end{array}\right| \begin{aligned}
& \cdot\left[\left(\lambda-\left(b_{1}+c_{1} \cdot i\right)\right) \cdot\left(\lambda-\left(b_{1}-c_{1} \cdot i\right)\right)\right]^{m_{1}} \cdot \\
& \cdot\left[\left(\lambda-\left(b_{2}+c_{2} \cdot i\right)\right) \cdot\left(\lambda-\left(b_{2}-c_{2} \cdot i\right)\right)\right]^{m_{2}} \cdot \ldots \cdot \\
& \cdot\left[\left(\lambda-\left(b_{p}+c_{p} \cdot i\right)\right) \cdot\left(\lambda-\left(b_{p}-c_{p} \cdot i\right)\right)\right]^{m_{p}}
\end{aligned}
$$

where $n_{1}+n_{2}+\ldots+n_{q}+2 \cdot m_{1}+2 \cdot m_{2}+\ldots+2 \cdot m_{p}=n$. In other words, we will assume that the characteristic equation (6) attached to the system (4) has $q$ real and distinct solutions with multiplicity orders $n_{1}, n_{2}, \ldots$, respectively $n_{q}$ and $2 \cdot p$ distinct conjugate complex solutions with multiplicity orders $m_{1}, m_{2}, \ldots$, respectively $m_{p}$.

We will assume that the free terms $f_{i}(x)$, $i \in \overline{1, n}$ are functions in the form of

$$
\begin{aligned}
f_{i 1}(x) \cdot e^{\alpha_{i 1} \cdot x}+P_{i 2}(x) \cdot e^{\alpha_{i 2} \cdot x}+\ldots+P_{i k_{i}}(x) \cdot e^{\alpha_{i k_{i}} \cdot x}+ \\
+e^{\gamma_{i 1} \cdot x} \cdot\left(Q_{i 1}(x) \cdot \cos \left(\beta_{i 1} \cdot x\right)+R_{i 1}(x) \cdot \sin \left(\beta_{i 1} \cdot x\right)\right)+ \\
+e^{\gamma_{i 2} \cdot x} \cdot\left(Q_{i 2}(x) \cdot \cos \left(\beta_{i 2} \cdot x\right)+R_{i 2}(x) \cdot \sin \left(\beta_{i 2} \cdot x\right)\right)+. .+ \\
+e^{\gamma_{i l_{i}} \cdot x} \cdot\left(Q_{i l_{i}}(x) \cdot \cos \left(\beta_{i l_{i}} \cdot x\right)+R_{i l_{i}}(x) \cdot \sin \left(\beta_{i l_{i}} \cdot x\right)\right)
\end{aligned}
$$

where $k_{i}$ and $l_{i}, i \in \overline{1, n}$ are natural numbers (positive integers), $\alpha_{i j}, i \in \overline{1, n}$ and $j \in \overline{1, k_{i}}$ and $\beta_{i j}, \quad \gamma_{i j}, i \in \overline{1, n}$ and $j \in \overline{1, r_{i}}$ are real numbers, and $P_{i j}, i \in \overline{1, n}$ and $j \in \overline{1, k_{i}}$ and $Q_{i j}, R_{i j}, i \in \overline{1, n}$ and $j \in \overline{1, r_{i}}$ are polynomials with real coefficients. 
We also assume that $\alpha_{i_{1} j_{1}}, \alpha_{i_{1} j_{2}}, \ldots, \alpha_{i_{1} j_{\eta}}$, $i_{a} \in \overline{1, n}$ and $j_{a} \in \overline{1, k_{i_{a}}}$, with $a \in \overline{1, r}$, and $\gamma_{p_{1} q_{1}}+\beta_{p_{1} q_{1}} \cdot i, \quad \gamma_{p_{2} q_{2}}+\beta_{p_{2} q_{2}} \cdot i$ $\gamma_{p_{s} q_{s}}+\beta_{p_{s} q_{s}} \cdot i, \quad p_{b} \in \overline{1, n} \quad$ and $\quad q_{b} \in \overline{1, l_{p_{b}}}$, with $b \in \overline{1, s}$ are among the solutions of the characteristic equation (5) which has the expansion (6).

Under these circumstances, a particular solution of the linear differential equation system with constant coefficients (3), where the free terms $f_{i}(x), i \in \overline{1, n}$, are like (7) will be as

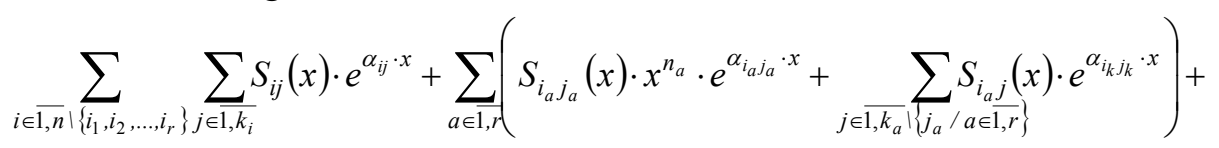

$$
\begin{aligned}
& y_{i p}(x)=+\sum_{i \in \overline{1, n} \mid\left\{p_{1}, p_{2}, \ldots, p_{l}\right\}} \sum_{j \in \overline{1, l_{i}}} e^{\gamma_{i j} \cdot x} \cdot\left[U_{i j}(x) \cdot \cos \left(\beta_{i j} \cdot x\right)+V_{i j}(x) \cdot \sin \left(\beta_{i j} \cdot x\right)\right]+\quad, i \in \overline{1, n} \\
& +\sum_{b \in 1, s}\left\{x^{n_{b}} \cdot e^{\gamma_{p b q b} \cdot x} \cdot\left[U_{p_{b} q_{b}}(x) \cdot \cos \left(\beta_{p_{b} q_{b}} \cdot x\right)+V_{p_{b} q_{b}}(x) \cdot \sin \left(\beta_{p_{b} q_{b}} \cdot x\right)\right]+\right. \\
& +\sum_{j \in \overline{1, l_{b}}}\left\{e_{\left\{q_{b} / b \in \overline{1, s}\right\}} e^{\gamma_{p_{b} q_{b}} \cdot x} \cdot\left[U_{p_{b} q_{b}}(x) \cdot \cos \left(\beta_{p_{b} q_{b}} \cdot x\right)+V_{p_{b} q_{b}}(x) \cdot \sin \left(\beta_{p_{b} q_{b}} \cdot x\right)\right]\right\}
\end{aligned}
$$

where $S_{i j}, i \in \overline{1, n}$ and $j \in \overline{1, k_{i}}$ and $U_{i j}, V_{i j}$, $i \in \overline{1, n}$ and $j \in \overline{1, l_{i}}$ are polynomials with degree $S_{i j}=$ degree $P_{i j}, i \in \overline{1, n}$ and $j \in \overline{1, k_{i}}$ and degree $U_{i j}=$ degree $V_{i j}=\max \{$ degree $Q_{i j}$, degree $\left.R_{i j}\right\}, i \in \overline{1, n}$ and $j \in \overline{1, l_{i}}$, and $n_{a}$ is the multiplicity order of the radical $\alpha_{i_{a} j_{a}}$, $a \in \overline{1, r}$ and $n_{b}$ is the multiplicity order of the radical $\gamma_{p_{b} q_{b}}+\beta_{p_{b} q_{b}} \cdot i, b \in \overline{1, s}$.

The form of the solution (7) considers the form of functions $f_{i}(x), i \in \overline{1, n}$ and the form of the characteristic equation (6).

Further on, we will illustrate the way in which the previously described method should be applied.
Let there be the linear differential equation system with constant coefficients

$$
\left\{\begin{array}{l}
y_{1}^{\prime}(x)=-y_{2}(x)+\cos x \\
y_{2}^{\prime}(x)=y_{1}(x)+\quad \sin 2 \cdot x
\end{array} .\right.
$$

We will first determine the solutions of the attached characteristic equation:

$$
\left|\begin{array}{cc}
-\lambda & -1 \\
1 & -\lambda
\end{array}\right|=\lambda^{2}+1=0
$$

The solutions of these equations are $\lambda_{1}, \lambda_{2}= \pm i$. Given the form of the constant terms and radicals of the characteristic equation, we will search for a particular solution such as

$$
\left\{\begin{array}{l}
y_{1 p}(x)=C_{1} \cdot \sin x+C_{2} \cdot \cos x+C_{3} \cdot x \cdot \sin x+C_{4} \cdot x \cdot \cos x+C_{5} \cdot \sin 2 \cdot x+C_{6} \cdot \cos 2 \cdot x \\
y_{2 p}(x)=C_{7} \cdot \sin x+C_{8} \cdot \cos x+C_{9} \cdot x \cdot \sin x+C_{10} \cdot x \cdot \cos x+C_{11} \cdot \sin 2 \cdot x+C_{12} \cdot \cos 2 \cdot x
\end{array} .\right.
$$

We have:

$$
\left\{\begin{array}{rl}
y_{1 p}^{\prime}(x)= & C_{1} \cdot \cos x-C_{2} \cdot \sin x+C_{3} \cdot \sin x+C_{3} \cdot x \cdot \cos x+C_{4} \cdot \cos x-C_{4} \cdot x \cdot \sin x+ \\
& +2 \cdot C_{5} \cdot \cos 2 \cdot x-2 \cdot C_{6} \cdot \sin 2 \cdot x \\
y_{2 p}^{\prime}(x)= & C_{7} \cdot \cos x-C_{8} \cdot \sin x+C_{9} \cdot \sin x+C_{9} \cdot x \cdot \cos x+C_{10} \cdot \cos x-C_{10} \cdot x \cdot \sin x+ \\
& +2 \cdot C_{11} \cdot \cos 2 \cdot x-2 \cdot C_{12} \cdot \sin 2 \cdot x
\end{array} .\right.
$$

Introducing the functions $y_{1 p}$ and $y_{2 p}$ and

their derivatives in the system (8) we get: 
$C_{1} \cdot \cos x-C_{2} \cdot \sin x+C_{3} \cdot \sin x+C_{3} \cdot x \cdot \cos x+C_{4} \cdot \cos x-C_{4} \cdot x \cdot \sin x+2 \cdot C_{5} \cdot \cos 2 \cdot x-2 \cdot C_{6} \cdot \sin 2 \cdot x=$ $=-\left(C_{7} \cdot \sin x+C_{8} \cdot \cos x+C_{9} \cdot x \cdot \sin x+C_{10} \cdot x \cdot \cos x+C_{11} \cdot \sin 2 \cdot x+C_{12} \cdot \cos 2 \cdot x\right)+\cos x$

$C_{7} \cdot \cos x-C_{8} \cdot \sin x+C_{9} \cdot \sin x+C_{9} \cdot x \cdot \cos x+C_{10} \cdot \cos x-C_{10} \cdot x \cdot \sin x+2 \cdot C_{11} \cdot \cos 2 \cdot x-2 \cdot C_{12} \cdot \sin 2 \cdot x=$ $=C_{1} \cdot \sin x+C_{2} \cdot \cos x+C_{3} \cdot x \cdot \sin x+C_{4} \cdot x \cdot \cos x+C_{5} \cdot \sin 2 \cdot x+C_{6} \cdot \cos 2 \cdot x+\sin 2 \cdot x$

By identifying the coefficients, we obtain

the follwing system of linear equations:

$$
\left\{\begin{array}{l}
C_{1}+C_{4}=-C_{8}+1 \\
-C_{2}+C_{3}=-C_{7} \\
C_{3}=-C_{10}
\end{array},\left\{\begin{array}{l}
-C_{4}=-C_{9} \\
2 \cdot C_{5}=-C_{12} \\
-2 \cdot C_{6}=-C_{11}
\end{array},\left\{\begin{array} { l } 
{ C _ { 7 } + C _ { 1 0 } = C _ { 2 } } \\
{ - C _ { 8 } + C _ { 9 } = C _ { 1 } } \\
{ C _ { 9 } = C _ { 4 } }
\end{array} \text { and } \left\{\begin{array}{l}
-C_{10}=C_{3} \\
2 \cdot C_{11}=C_{6} \\
-2 \cdot C_{12}=C_{5}+1
\end{array} .\right.\right.\right.\right.
$$

The solution of the above system is:

$$
\left\{\begin{array} { l } 
{ C _ { 1 } = 1 / 2 } \\
{ C _ { 2 } = C _ { 7 } = 0 } \\
{ C _ { 3 } = C _ { 1 0 } = 0 } \\
{ C _ { 4 } = C _ { 9 } = 1 / 2 }
\end{array} \text { and } \left\{\begin{array}{l}
C_{5}=1 / 3 \\
C_{6}=C_{11}=0 \\
C_{8}=0 \\
C_{12}=-2 / 3
\end{array} .\right.\right.
$$

After determining the particular solution, we may write the general solution of the given system.

Further on, we will assume that we have a system of linear differential equations with constant coefficients such as:

$$
\left\{\begin{array}{l}
y_{1}^{\prime}(x)=-y_{2}(x)+e^{2 \cdot x} \cdot(2 \cdot x+1) \\
y_{2}^{\prime}(x)=y_{1}(x)+\quad e^{3 \cdot x} \cdot\left(4 \cdot x^{2}-3 \cdot x+2\right)
\end{array}\right.
$$

In conclusion, a particular solution of the given system (8) is

$\left\{\begin{array}{l}y_{1 p}(x)=1 / 2 \cdot \sin x+1 / 2 \cdot x \cdot \cos x+1 / 3 \cdot \sin 2 \cdot x \\ y_{2 p}(x)=1 / 2 \cdot x \cdot \sin x-2 / 3 \cdot \cos 2 \cdot x\end{array}\right.$.

Given that system (10) has the same characteristic equation (9) and that $\lambda=2$ and $\lambda=3$ are not its solutions, we will try to find a particular solution of the system, such as:

$$
\left\{\begin{array}{l}
y_{1 p}(x)=e^{2 \cdot x} \cdot\left(A_{1} \cdot x+B_{1}\right)+e^{3 \cdot x} \cdot\left(C_{1} \cdot x^{2}+D_{1} \cdot x+E_{1}\right) \\
y_{2 p}(x)=e^{2 \cdot x} \cdot\left(A_{2} \cdot x+B_{2}\right)+e^{3 \cdot x} \cdot\left(C_{2} \cdot x^{2}+D_{2} \cdot x+E_{2}\right)
\end{array} .\right.
$$

We have:

$$
\left\{\begin{array}{l}
y_{1 p}^{\prime}(x)=e^{2 \cdot x} \cdot\left(2 \cdot A_{1} \cdot x+A_{1}+2 \cdot B_{1}\right)+e^{3 \cdot x} \cdot\left[3 \cdot C_{1} \cdot x^{2}+\left(2 \cdot C_{1}+3 \cdot D_{1}\right) \cdot x+D_{1}+3 \cdot E_{1}\right] \\
y_{2 p}^{\prime}(x)=e^{2 \cdot x} \cdot\left(2 \cdot A_{2} \cdot x+A_{2}+2 \cdot B_{2}\right)+e^{3 \cdot x} \cdot\left[3 \cdot C_{2} \cdot x^{2}+\left(2 \cdot C_{2}+3 \cdot D_{2}\right) \cdot x+D_{2}+3 \cdot E_{2}\right]
\end{array} .\right.
$$

Introducing functions $y_{1 p}$ and $y_{2 p}$ and their derivatives in the system (10) we get:

$$
\left\{\begin{array}{l}
e^{2 \cdot x} \cdot\left(2 \cdot A_{1} \cdot x+A_{1}+2 \cdot B_{1}\right)+e^{3 \cdot x} \cdot\left[3 \cdot C_{1} \cdot x^{2}+\left(2 \cdot C_{1}+3 \cdot D_{1}\right) \cdot x+D_{1}+3 \cdot E_{1}\right]= \\
=-\left[e^{2 \cdot x} \cdot\left(A_{2} \cdot x+B_{2}\right)+e^{3 \cdot x} \cdot\left(C_{2} \cdot x^{2}+D_{2} \cdot x+E_{2}\right)\right]+e^{2 \cdot x} \cdot(2 \cdot x+1) \\
e^{2 \cdot x} \cdot\left(2 \cdot A_{2} \cdot x+A_{2}+2 \cdot B_{2}\right)+e^{3 \cdot x} \cdot\left[3 \cdot C_{2} \cdot x^{2}+\left(2 \cdot C_{2}+3 \cdot D_{2}\right) \cdot x+D_{2}+3 \cdot E_{2}\right]= \\
=e^{2 \cdot x} \cdot\left(A_{1} \cdot x+B_{1}\right)+e^{3 \cdot x} \cdot\left(C_{1} \cdot x^{2}+D_{1} \cdot x+E_{1}\right)+e^{3 \cdot x} \cdot\left(4 \cdot x^{2}-3 \cdot x+2\right)
\end{array}\right.
$$

By identifying the coefficients, we obtain

the following linear equation system: 


$$
\left\{\begin{array} { l } 
{ 2 \cdot A _ { 1 } = - A _ { 2 } + 2 } \\
{ A _ { 1 } + 2 \cdot B _ { 1 } = - B _ { 2 } + 1 } \\
{ 3 \cdot C _ { 1 } = - C _ { 2 } } \\
{ 2 \cdot C _ { 1 } + 3 \cdot D _ { 1 } = - D _ { 2 } } \\
{ D _ { 1 } + 3 \cdot E _ { 1 } = - E _ { 2 } }
\end{array} \text { and } \left\{\begin{array}{l}
2 \cdot A_{2}=A_{1} \\
A_{2}+2 \cdot B_{2}=B_{1} \\
3 \cdot C_{2}=C_{1}+4 \\
2 \cdot C_{2}+3 \cdot D_{2}=D_{1}-3 \\
D_{2}+3 \cdot E_{2}=E_{1}+2
\end{array} .\right.\right.
$$

The solution of the above system is:

$$
\left\{\begin{array}{l}
A_{1}=4 / 5 \\
A_{2}=2 / 5
\end{array},\left\{\begin{array}{l}
B_{1}=4 / 25 \\
B_{2}=-3 / 25
\end{array},\left\{\begin{array}{l}
C_{1}=-2 / 5 \\
C_{2}=6 / 5
\end{array},\left\{\begin{array} { l } 
{ D _ { 1 } = 3 9 / 5 0 } \\
{ D _ { 2 } = - 7 7 / 5 0 }
\end{array} \text { and } \left\{\begin{array}{l}
E_{1}=-294 / 500 \\
E_{2}=492 / 500
\end{array}\right.\right. \text {. }\right.\right.\right.
$$

In conclusion, a particular solution of the given system (10) is

$$
\left\{\begin{array}{l}
y_{1 p}(x)=(4 / 5 \cdot x+4 / 25) \cdot e^{2 \cdot x}+\left(-2 / 5 \cdot x^{2}+39 / 50 \cdot x-294 / 500\right) \cdot e^{3 \cdot x} \\
y_{2 p}(x)=(2 / 5 \cdot x-3 / 25) \cdot e^{2 \cdot x}+\left(6 / 5 \cdot x^{2}-77 / 50 \cdot x+492 / 500\right) \cdot e^{3 \cdot x}
\end{array} .\right.
$$

After determining the particular solution, we may write the general solution of the given system.

Finally, let us assume that we now have the following system of linear differential equations with constant coefficients:

$$
\left\{\begin{array}{l}
y_{1}^{\prime}(x)=\quad-y_{2}(x)+e^{x} \cdot(2 \cdot x+1) \\
y_{2}^{\prime}(x)=y_{1}(x)+\quad 4 \cdot x^{2}-3 \cdot x+2
\end{array}\right. \text {. }
$$

Since the system (11) has the same characteristic equation (9) and that $\lambda=0$ and $\lambda=1$ are not its solutions, we will try to find a particular solution of the system in the form of:

$$
\left\{\begin{array}{l}
y_{1 p}(x)=e^{x} \cdot\left(A_{1} \cdot x+B_{1}\right)+C_{1} \cdot x^{2}+D_{1} \cdot x+E_{1} \\
y_{2 p}(x)=e^{x} \cdot\left(A_{2} \cdot x+B_{2}\right)+C_{2} \cdot x^{2}+D_{2} \cdot x+E_{2}
\end{array} .\right.
$$

We have:

$$
\left\{\begin{array}{l}
y_{1 p}^{\prime}(x)=e^{x} \cdot\left(A_{1} \cdot x+A_{1}+B_{1}\right)+2 \cdot C_{1} \cdot x+D_{1} \\
y_{2 p}^{\prime}(x)=e^{x} \cdot\left(A_{2} \cdot x+A_{2}+B_{2}\right)+2 \cdot C_{2} \cdot x+D_{2}
\end{array} .\right.
$$

Introducing the functions $y_{1 p}$ and $y_{2 p}$ and their derivatives in the system (11) we get:

$\left\{\begin{array}{l}e^{x} \cdot\left(A_{1} \cdot x+A_{1}+B_{1}\right)+2 \cdot C_{1} \cdot x+D_{1}=-\left[e^{x} \cdot\left(A_{2} \cdot x+B_{2}\right)+C_{2} \cdot x^{2}+D_{2} \cdot x+E_{2}\right]+e^{x} \cdot(2 \cdot x+1) \\ e^{x} \cdot\left(A_{2} \cdot x+A_{2}+B_{2}\right)+2 \cdot C_{2} \cdot x+D_{2}=e^{x} \cdot\left(A_{1} \cdot x+B_{1}\right)+C_{1} \cdot x^{2}+D_{1} \cdot x+E_{1}+4 \cdot x^{2}-3 \cdot x+2\end{array}\right.$

By identifying the coefficients, we obtain the following linear equations system:

$$
\left\{\begin{array} { l } 
{ A _ { 1 } = - A _ { 2 } + 2 } \\
{ A _ { 1 } + B _ { 1 } = - B _ { 2 } + 1 } \\
{ 2 \cdot C _ { 1 } = - D _ { 2 } } \\
{ - C _ { 2 } = 0 } \\
{ D _ { 1 } = - E _ { 2 } }
\end{array} \text { and } \left\{\begin{array}{l}
A_{2}=A_{1} \\
A_{2}+B_{2}=B_{1} \\
2 \cdot C_{2}=D_{1}-3 \\
D_{2}=E_{1}+2 \\
C_{1}+4=0
\end{array} .\right.\right.
$$

The solution of the above system is:

$$
\left\{\begin{array} { l } 
{ A _ { 1 } = 1 } \\
{ A _ { 2 } = 1 } \\
{ B _ { 1 } = 1 / 2 } \\
{ B _ { 2 } = - 1 / 2 } \\
{ C _ { 1 } = - 4 }
\end{array} \text { and } \left\{\begin{array}{l}
C_{2}=0 \\
D_{1}=3 \\
D_{2}=8 \\
E_{1}=6 \\
E_{2}=-3
\end{array} .\right.\right.
$$

In conclusion, a particular solution of the given system (10) is

$$
\left\{\begin{array}{l}
y_{1 p}(x)=e^{x} \cdot(x+1 / 2)-4 \cdot x^{2}+3 \cdot x+6 \\
y_{2 p}(x)=e^{x} \cdot(x-1 / 2)+8 \cdot x-3
\end{array} .\right.
$$

After determining the particular solution, we may write the general solution of the given system. 


\section{Conclusions and disscussions}

Determining a particular solution for a system of linear differential equations with constant coefficients like (3), having the free terms $f_{i}(x), i \in \overline{1, n}$, such as (2), can be done generally, regardless of the form of the characteristic equation (5), as we have seen in the case of $n$-th order linear differential equations with constant coefficients.

It is a simple and efficient method for determining a particular solution without neither having to determine all solutions to the characteristic equation - in the case of the variation of constants method, nor a series of calculations that involve successive elimination as in the case of the cancellation method to solving $\mathrm{n}$-th order linear differential equations with constant coefficients.

Moreover, for the variation of constants method, determining all solutions of the characteristic equation is generally very complicated, and determining a particular solution additionally requires replacing the general solution in the system and then determining the functions generated by constants, which involves, besides solving an $n$-th order system, integrating $n$ functions. The method presented actually builds the particular solution and ultimately resumes only to determining the solution of a system of linear equations. 